\title{
Prevalência de Stress e Fontes Estressoras em Estudantes do Último Período do Curso de Odontologia da Universidade de Gurupi-TO.
}

\section{Prevalence of Stress and Stress Sources in students of the Last period of the Course of Odontology of the University of Gurupi-TO}

Angelo Martin Colmenares Añaguari', Lukas Vargas Lindemaier², Vinicius Lopes Marinho ${ }^{3}$ Jeann Bruno Ferreira da Silva, ${ }^{4}$

\section{RESUMO}

O estresse é considerado quando o indivíduo passa por algumas circunstâncias que fazem com que ele reaja com fortes tensões, alterando o ritmo do funcionamento do sistema orgânico, ameaçando o bem estar físico e mental dos indivíduos Nessa perspectiva, o estudo objetivou identificar a prevalência de estresse em estudantes do último período do curso de odontologia da Universidade Unirg de 2018/2, bem como constatar a fase do estresse, manifestações dos sintomas, identificar as fontes estressoras para os acadêmicos. Os dados coletados foram obtidos através da aplicação do inventário de sintomas de Stress para adultos de Lipp (ISSL), para constatar a presença de estresse e identificação das fases e sintomas existentes nos participantes do estudo, além de um questionário para levantamento dos estressores. Dos resultados obtidos do total da amostragem mostram que os estudantes se encontram estressados e predominância da fase de resistência 93\%, fase de alerta $7 \%$ e nenhuma na fase de exaustão e quase-exaustão, dos sintomas destacou 0 psicológico 64\%, quanto às fontes de estresse dos 16 itens a maior prevalência foram: ansiedade em relação ao futuro da profissão, estar longe da família, relacionamento com os Professores, falta de recursos e materiais da Instituição, muitas atividades acadêmicas e estresse associado a gestão do tempo. Conclui-se que a alta ocorrência de estresse nos formandos se integra as exigências que competem a eles no presente e futuro profissional.

Descritores: Estresse, Estressores, odontologia.

\section{ABSTRACT}

Stress is considered when the individual undergoes some circumstances that cause him to react with strong tensions, altering the rhythm of the functioning of the organic system, threatening the physical and mental well-being of the individuals. In this perspective, the study aimed to identify the prevalence of stress in students of the last period of the course of dentistry of University Unirg of 2018/2, as well as to verify the phase of the stress, manifestations of the symptoms, to identify the sources stressors for the academics. The collected data were obtained by the population researched through the application of the Stress Symptom Inventory for Adults of Lipp (ISSL), to verify the presence of stress and identification of the phases and symptoms prevailed in the participants of the study and a questionnaire to survey the stressors. Results from the total sample showed that students were stressed and predominance of the resistance phase $93 \%$, alert phase $7 \%$ and none in the phase of exhaustion and quasiexhaustion, of the symptoms emphasized the psychological 64\%, regarding the sources of stress of the 16 items the highest prevalence were: anxiety about the future of the profession, being away from the family, relationship with the Teachers, lack of resources and materials of the Institution, many academic activities and stress associated with time management. It is concluded that the high occurrence of stress in the trainees integrates the requirements that compete for them in the present and future professional.

Descriptors: Stress, Stressors, dentistry. 


\section{INTRODUÇĀO}

Durante a vida acadêmica ocorrem inúmeras mudanças no estilo e qualidade de vida dos estudantes. Alguns fatores como novos grupos sociais, aglomerar-se com estranhos, morar longe dos pais, pressões acadêmicas, frustrações, uso de bebidas alcoólicas, tabagismo, angustias e a grande busca de equilíbrio dos compromissos sociais são fatores que desencadeiam distúrbios patológicos gerando stress. ${ }^{1}$

O stress é um conjunto de manifestações físicas e psicológicas diante de circunstâncias que obriga a pessoa se adaptar, seja num sentindo de um fato ou à condições formidáveis. ${ }^{2}$

Cabe aqui ressaltar que o presente trabalho utilizará o termo stress que provém do inglês "stressors", termo bastante utilizado na física e engenharia para determinar forças que atuam contra determinada resistência ao invés do termo stress que resulta de uma adaptação para a língua portuguesa. ${ }^{2}$

A tendência a se estressar está ligada a distorções cognitivas, ou seja, pensamentos errados ou distorcidos, avaliações interpretações erradas acerca de eventos da vida. Além disso algumas pessoas são biologicamente propensas a se estressarem e essa tendência e chamada de vulnerabilidade.Todos os indivíduos quando estão estressados o organismo trabalha pra restabelecer o equilíbrio eliminando o estresse não havendo a resposta o organismo começa a se desgastar ficando vulnerável a doenças. ${ }^{1}$

De acordo com a autora acima, no stress, o organismo precisa lidar com situações que exijam um esforço emocional para serem superadas, seja para uma situação positiva ou negativa, sendo o stress uma dessa reações. Envolve componentes físicos, psicológicos, hormonais e mentais. Se desenvolve em 4 fases, sendo elas a fase do alerta, fase de resistência, fase da quase exaustão e a fase de exaustão. ${ }^{2}$

O stress pode influenciar esses fatores pois gera uma grande cobrança de responsabilidades a serem cumpridas na vivência acadêmica e profissional. ${ }^{2}$

Dentre os diversos cursos superiores, destaca-se o curso de Odontologia que se caracteriza por possuir uma extensa carga horária teórica e prática, que influencia diretamente na qualidade de vida dos estudantes. Os Períodos de passagem do ambiente acadêmico para o pré-clínico(treinamento), em seguida para o clinico e definitivamente ao ambiente de trabalho, causam preocupações, habilidades profissionais e pressão advinda do trabalho que contribuem negativamente sobre a saúde dos acadêmicos. ${ }^{3}$ 
Estudos com o propósito de investigar o stress em cursos da área da saúde evidenciaram uma prevalência de stress em estudantes de odontologia, podendo chegar a um valor de até $49,7 \%$ justificam o motivo da investigação no curso de Odontologia da I.E.S. ${ }^{4}$

Diante do exposto acima, questionou-se qual a prevalência de stress em estudantes do último período do curso de Odontologia da Universidade de Gurupi/UNIRG e quais os fatores estressores?

Desta maneira o presente estudo teve como objetivo investigar prevalência de stress em estudantes do último período do curso de Odontologia da Universidade UNIRG e os fatores estressores, bem como constatar em que fase o stress se encontra; verificar a predominância dos sintomas (físicos ou psicológicos) e conhecer as fontes estressoras para os estudantes.

\section{MATERIAIS E METODOS}

Tratou-se de uma pesquisa de campo, quantitativo-descritiva, realizada com 22acadêmicos do último período do curso de Odontologia, em 2018/2. A mesma foi aprovada por um Comitê de Ética em Pesquisa com Seres Humanos, conforme parecer ํo 2.680.737.

Utilizou-se como critério de inclusão: alunos que estivessem devidamente matriculado no último período do curso de Odontologia em 2018/2, com previsão de concluir todas as disciplinas do semestre 2018.2 e que aceitassem participar da pesquisa. Já os de exclusão foram: alunos que estivessem com o curso trancado em 2018/2, que não concluísse todas as disciplinas do semestre 2018/2 e recusassem a participação na pesquisa. Como instrumento de coleta de dados, foi utilizado o Inventário de Sintomas de Stress para Adultos (I. S.S. L) ${ }^{2}$, bem como um questionário para levantamento das fontes estressoras. Cabe aqui, ressaltar que todas as etapas da pesquisa aconteceram na sala do $10^{\circ}$ período do curso de psicologia do Centro Universitário Unirg, foi preparada especialmente para a aplicação do Inventário de Sintomas de Stress para Adultos de Lipp.

Os participantes foram divididos em grupo de no máximo 20 pessoas, conforme determina o manual do presente inventário. Para a análise será utilizado método quantitativo, onde será efetuada análise descritiva dos dados (freqüência e porcentagem) através do software SPSS - StatisticalPackage for the Social Sciencesversão 20.0 para 
Windows. No qual os dados serão apresentados em números absolutos e relativos e demonstrados em gráficos e tabelas.

\section{RESULTADOS E DISCUSSAOO}

Tabela 1. Caracterização dos Participantes

\begin{tabular}{lcc}
\hline Variáveis & n. & $\%$ \\
\hline Sexo & 09 & $41 \%$ \\
Masculino & 13 & $59 \%$ \\
Feminino & & \\
Faixa etária & 16 & $72 \%$ \\
20 a 25 anos & 05 & $23 \%$ \\
26 a 30 anos & 01 & $5 \%$ \\
Acima de 30 anos & & \\
Estado Civil & 20 & $91 \%$ \\
Solteiro & 02 & $9 \%$ \\
Casado & & \\
\hline
\end{tabular}

$\mathrm{De}$ acordo com a tabela 1 os sujeitos encontrados na pesquisa foram maioria do sexo feminino $59 \%$ (13) contra $41 \%$ (9) do sexo masculino, justificando o resultado do estresse ter prevalecido nas mulheres, vindo de encontro com a pesquisa que encontrou as manifestações de estresse em maior número no gênero feminino do que no masculino, as explicações para o evento é que as mulheres podem ser mais vulneráveis ao acometimento do estresse, podem ser mais francas em admitir o estresse ou de fato podem sofrerem mais em relação ao sexo oposto. $^{5}$

A maioria das acadêmicas se encontra na faixa etária de 20 a 25 anos $72 \%$, sendo a maior parte solteira $91 \%$. Tal fato também se deve por existir mais mulheres que homens no curso e conseqüentemente na pesquisa as mulheres prevaleceram em maior número. 


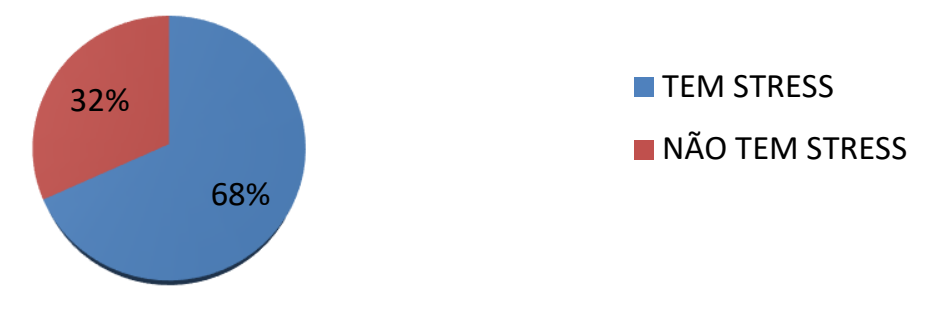

Fonte: Inventário de Sintomas de Stress para Adultos (LIPP, 2000) aplicado nos acadêmicos da Universidade Unirg da cidade de Gurupi-TO no mês de Novembro de 2018.

De acordo com o gráfico 1 verificou que os 22 graduandos que preencheram o Inventario de Sintomas de Stress de Lipp (ISSL), a maioria apresentaram sinais de estresse que corresponde a $68 \%$ do total da amostra, em comparação aos $32 \%$ que não apresentaram estresse. Por se tratar do último ano da graduação são acumuladas atividades acadêmicas e pessoais, além das preocupações que estão presentes durante e posterior a formação. Resultados esses que são concordantes com os encontrados na em pesquisa semelhante que demonstrou que $95,5 \%$ dos estudantes estavam com estresse devido as dificuldades em executar os fazeres da rotina universitária. ${ }^{6}$

Em relação a tal situação foi constatado a presença de estresse em estudantes do ensino superior. ${ }^{7}$ Ainda identificaram que $74 \%$ do total da sua amostra se encontram estressados, próximo ao valor obtido na presente pesquisa. ${ }^{8}$ Houve a constatação de que alunos dos últimos períodos apresentavam mais o estresse em relação aos iniciantes, motivados pelos fatores como obrigações a serem cumpridas nas atividades teórica e principalmente as práticas, exigência maior por parte de supervisores quanto as atribuições acadêmicas, apreensões relacionados ao mercado profissional e o trabalho de conclusão de curso.

Sujeitos que se assemelham com os da respectiva pesquisa. Ressalta-se a dimensão dos acontecimentos de estresse em inúmeros ambientes entre eles o contexto universitário onde o mesmo vem sendo debatido em diversas instâncias e áreas do conhecimento como na psicologia e entre outras. Há décadas em nosso país vem sendo desenvolvidos trabalhos destacando a relevância do assunto na sociedade Brasileira. ${ }^{9}$ 


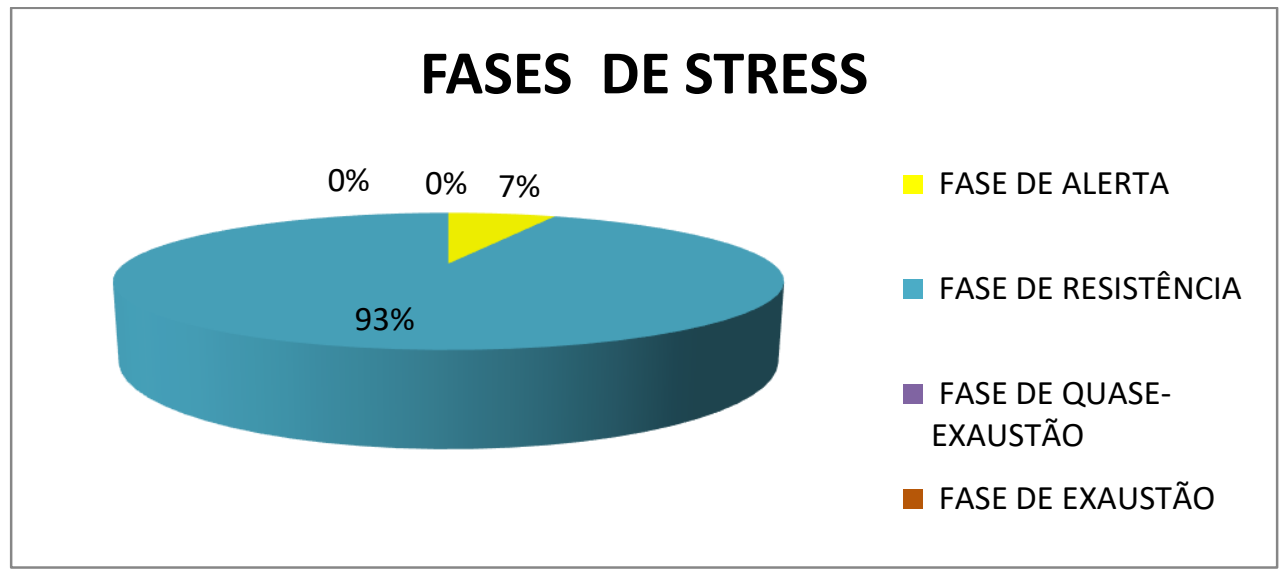

Fonte: Inventário de Sintomas de Stress para Adultos (LIPP, 2000) aplicado nos acadêmicos Universidade Unirg da cidade de Gurupi-TO no mês de Novembro de 2018.

Em referência as fases do estresse demonstrada no gráfico 2, dos estudantes que se encontram estressados a maior predominância entre os sujeito foi na fase de resistência com 93\%, concordando com a pesquisas a fase a qual predominou, resultados altos provenientes da rigidez do contexto acadêmico. ${ }^{10}$ Também confirmando, as descobertas da literatura de a fase de resistência a que mais se destacou. Nela a pessoa tenta restabelecer o equilíbrio orgânico despendida na fase de alerta pelo gasto de energia desnecessário para resistir ao estressor, com isso há uma maior probabilidade de adoecerem $^{11}$

Logo em seguida na pesquisa com acadêmicos que se encontram estressados não foram encontrados nenhum que apresentou característica sintomatológica que pudesse resultar na fase de quase exaustão $0 \%$, diferente do resultados encontrados na literatura onde verificou-se que apenas $4 \%$ com traços sintomatológico dessa fase. ${ }^{12}$

A fase de quase exaustão tem como sintomas característicos, o organismo manifestar sinais de deterioração e as defesas já não resisti às pressões e não conseguindo retornar ao equilíbrio interno, havendo momento de oscilação entre bem estar e desconforto, com isso os órgãos que são suscetíveis ao adoecimento pode ser afetado pelas doenças, não havendo controle do estresse pode passar para o outro estagio. Enquanto que na fase de exaustão os participantes não apresentaram níveis significativo de manifestações de estresse sendo também $0 \%$ se encontravam nessa fase, tal achado tem analogia com os encontrados em amostras coletadas em seu trabalho foi a que esteve em menos evidência.9 Nela os sintomas aparecem com uma maior intensidade há o desequilíbrio total da resistência orgânica, prevalecendo às 
enfermidades de ordem mais graves como doenças coronárias, gastrites, dermatites entre outras, o controle e gerenciamento do estresse devem ser feito com máxima urgência ${ }^{2}$

$\mathrm{Na}$ atual pesquisa com acadêmicos que se encontram estressados foram encontrados na fase de alerta com $7 \%$, concordando com o resultado que identificou que os sujeitos que foram submetidos ao teste (ISSL) em seu estudo 6,19\% possuíam a sintomatologia da fase de alerta. ${ }^{9}$ Considerando que a situação desgastante na qual se encontra os acadêmicos do último período de odontologia, véspera de conclusão do curso os afazeres a serem cumpridos são abundantes chegam a ultrapassar muita das vezes a capacidade de cumpri-las, assim, contribuindo para o desenvolvimento das fases de estresse considerada mais exaustivas/graves. Os sintomas manifestados entre os estudante avaliados na pesquisa, os de ordem psicológicas tiveram maior destaque $70 \%$ ultrapassando os de ordem física obteve $30 \%$, similar a descoberta feita, onde verificou que houve uma proeminência maior dos sintomas psíquicos na maioria dos participantes do estudo. ${ }^{11}$

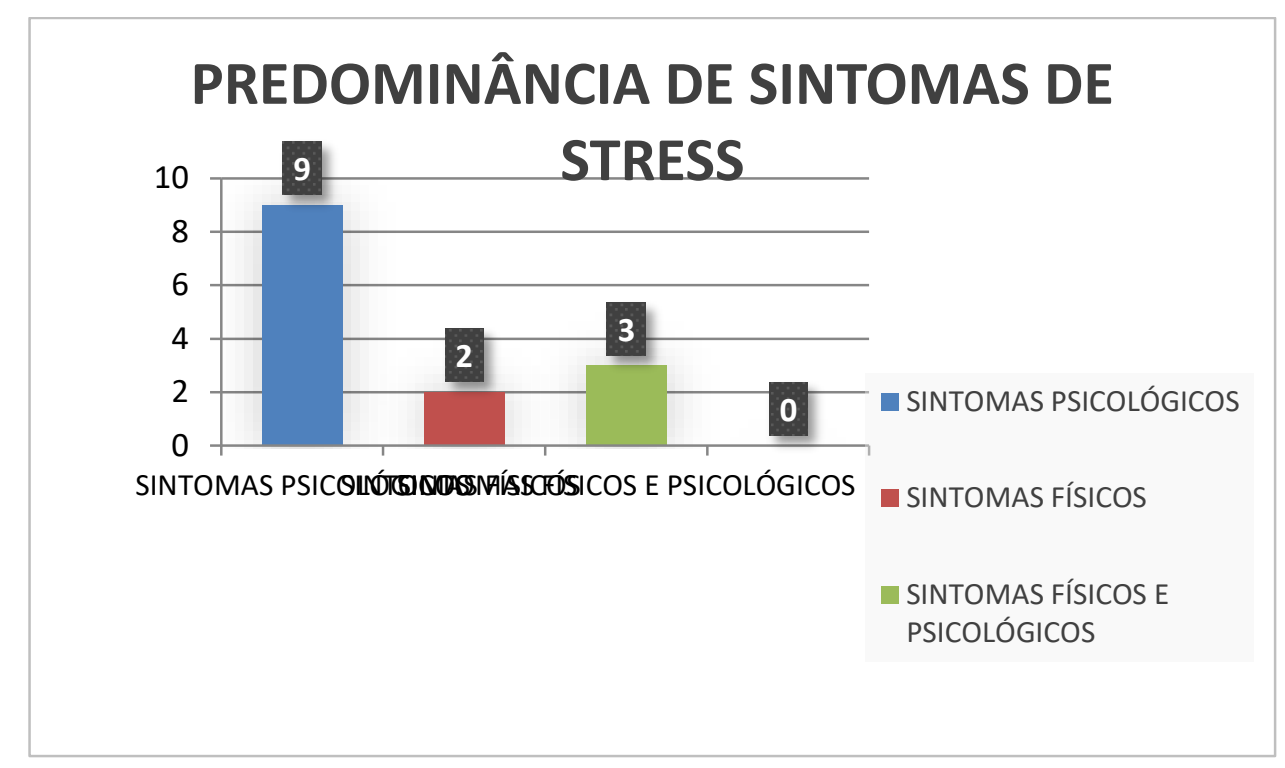

Fonte: Inventário de Sintomas de Stress para Adultos (LIPP, 2000) aplicado nos acadêmicos Universidade Unirg da cidade de Gurupi-TO no mês de Novembro de 2018.

Com relação à sintomatologia, 64\% desses alunos relataram predominância de sintomas psicológicos, seguidos de $14 \%$ com sintomas físicos e de $22 \%$ com sintomas físicos e psicológicos concomitantes. É importante ressaltar que a classificação de sintomas físicos e psicológicos como apresentados, em uma perspectiva integral do 
sujeito, não podem ser separados de forma estanque, posto o continuam entre as duas dimensões no sujeito. ${ }^{13}$

Tabela 2 - Fontes estressoras para os estudantes de odontologia

\begin{tabular}{ll}
\hline \multicolumn{1}{c}{ FONTES ESTRESSORAS } & N \\
\hline & 17 \\
Ansiedade em Relação ao futuro da profissão & 12 \\
Estar longe da família & 11 \\
Relacionamento com os Professores & 11 \\
Falta de recursos e materiais da Instituição & 10 \\
Muitas atividades acadêmicas & 10 \\
Estresse associado a gestão do tempo & \\
\hline
\end{tabular}

Fonte: Questionário elaborado pelo próprio pesquisador.

Para a análise dos resultados da tabela 2, foi considerada a menção do item pelo menos uma vez pelos graduandos, através dos questionários de 22 opções a serem marcada, porém dessas apenas seis consideradas pelo avaliado as principais, respondidos foi possível constatar as fontes estressoras para os alunos. Conforme ilustra a tabela 2, são várias as fontes estressoras no dia-a-dia do acadêmico de odontologia, diante disto, buscou-se elucidar os resultados que obteve-se do que pode ter gerado o estresse nos estudantes de odontologia. A partir de 22 questões apresentadas, 6 se destacaram. No que diz respeito as fontes geradoras de estresse, a ansiedade em relação ao futuro da profissão sobressaiu havendo maior destaque. ${ }^{14}$

Na pesquisa realizada com formandos de enfermagem, são mencionadas questões como: Expectativas e preocupações com o mundo do trabalho, a proximidade com término da formação estudantes apresentam preocupações, se vão conseguir ser inseridos no mercado de trabalho, ainda o medo em se vão conseguir desempenhar a função profissional a qual foi atribuída. ${ }^{3} \mathrm{O}$ trabalho de conclusão de curso aparece como sendo um dos fatores. Em seguida estar longe da família com 12 do total pesquisado. Tais fatores como relacionamento com professores e falta de recursos e materiais da instituição com 11; o fator familiar surge entre os estressores, como morar distante, as viagens para visitá-los podem repercutir em cansaços e atrapalhar o desempenho escolar e gerar estresse.9 Também baseado pelo autor, há uma discordância quando se trata de relações interpessoais de professor aluno, coordenação e aluno não citaram, enquanto 
que na presente houve a menção das questões de $1 \%$ aluno professor e $2 \%$ aluno coordenação. Já a relação aluno com aluno teve equivalência em ambas, foram citadas tanto na recente $1 \%$ quanto na do autor mencionado $18,2 \%$.

Em seguida o fator de muitas atividades acadêmicas 10; uma das questões de realce do estudo de $1168,3 \%$ dos avaliados referiram que a sobrecarga comprometeu o rendimento das atividades ofertadas pela instituição de ensino superior.

Em estudos realizados $73,5 \%$ consideraram as atividades acadêmicas sendo um fator estressante. ${ }^{12} \mathrm{Não}$ foram encontrados autores com pesquisa parecidas com estudantes que tivessem sido referenciadas quanto à avaliação de questões ligadas tais como: a falta de recursos materiais da instituição, e estresse associados à gestão de tempo para estar equiparando com o presente estudo.

\section{CONSIDERAÇOEES FINAIS}

A partir da pesquisa realizada foi possível constatar a ocorrência de estresse no contexto do curso de odontologia nos formandos do semestre 2018/2. A prevalência de estresse na turma do oitavo período de odontologia da Universidade Unirg foi alta, sendo que $68 \%$ dos participantes estão estressados.

Os resultados deste estudo constatam a presença das fases, a de resistência liderou o ranque identificada nos dados $93 \%$, o segundo lugar à fase de alerta com $7 \%$, e nenhuma nas fases de exaustão e quase-exaustão. Bem com os sintomas psicológicos em maioria dos participantes com $64 \%$, seguido de $22 \%$ dos físicos e psicológicos e $14 \%$ dos físicos. Tais levantamentos demonstram a vulnerabilidade em que se encontram os formandos do curso de odontologia ao acometimento do estresse e conseqüentemente ao adoecimento.

Com relação ás fontes estressoras foi possível identificar que as principais foram a ansiedade em Relação ao futuro da profissão, estar longe da família, relacionamento com os Professores, falta de recursos e materiais da Instituição, muitas atividades acadêmicas e estresse associado a gestão do tempo foram às seis que obteve resultados expressivos quando comparados com as outras. Para os respondentes da respectiva pesquisa foram a que consideram entre as principais fontes de estresse.

Cabe aqui ressaltar a necessidade de mais pesquisas sobre assunto em questão, porém já enfatizado em outros estudos, precisa ser mais ainda discutido no meio acadêmico. Não só discutir mais criar programas para a prevenção e até mesmo o 
enfrentamento do estresse nos alunos do curso, pois com certeza tal situação interfere no rendimento dos alunos e conseqüentemente na qualidade do ensino ofertado.

\section{REFERÉNCIAS}

1. LIIP, M. E. N. Manual do inventário de sintomas de stress para adultos de Lipp. São Paulo: Casa do Psicólogo; 2000.

2. LIIP, M.E.N., \& MALAGRIS, L. E. N. O stress emocional e seu tratamento. In B. Rangé (Org.), Psicoterapias cognitivo-comportamentais: um diálogo com a psiquiatria; Porto Alegre: Artmed; 2001. p.475490.

3. SILVA, V. L. S. et al. Fatores de Estresse no último Ano do Curso de Graduação em Enfermagem: Percepção dos Estudantes. Ver. Enferm. UERJ, Rio de Janeiro; 2011. p.121-126.

4. SPIGER, V. Fatores estressores entre estudantes do Curso de Graduação em Odontologia da UFSC. Florianópolis (SC): Universidade Federal de Santa Catarina; 2015.

5. TORQUATO, J. A. et al. Avaliação do estresse em estudantes universitários. Revista Científica Internacional Indexada ISSN 1679 9844, v. 3, n.14; 2010. p.140-154.

6. RIBEIRO, C. B. et al. O Estresse do Graduando de Enfermagem no Âmbito da Universidade. NEUROBIOLOGIA, Franca, v.74, n. 2; 2010.

7. MILSTED, J. G.; AMORIM, C.; SANTOS, M. Nível de Estresse em Alunos de Psicologia do Período Noturno. IX Congresso Nacional de Educação-EDUCERE III encontro Sul Brasileiro de Psicopedagogia da PUC-PR; 2009.

8. MONDARDO, A. H.; PEDON, E. A. Estresse e Desempenho Acadêmico em Estudantes Universitários. Revista de Ciências Humanas. Porto Alegre; 2007. p.1-21.

9. CALAIS, S. L. et al. Stress entre calouros e veteranos de jornalismo. Campinas, v. 24, n.1; 2007. p. 69-77.

Estudos de Psicologia,

10. CONTI, F. D.; JÚNIOR, A. R. Personalidade e rigor acadêmico: variáveis facilitadoras do surgimento do stress em alunos quintanistas de psicologia. Rev. ciênc. hum, Taubaté, v.12, n.1; 2006. p.33-49.

11. BONIFÁCIO, S. P. et al. Investigação e manejo de eventos estressores entre estudantes de Psicologia. Revista Brasileira de Terapias Cognitivas. V.7, n.1; 2011. p. 15-20.

12. AGUIAR, S. M. et al. Prevalência de sintomas de estresse nos estudantes de medicina. J BrasPsiquiatr, v.58, n. $1 ; 2009$. p. 34-38.

13. ASSIS, C. L, SILVA A. P. F, LOPES, M. S, SILVA, P. C. B, SANTINI, T. O. Sintomas de estresse em concluintes do curso de psicologia de uma faculdade privada do norte do País. Mudanças - Psicologia da Saúde, 21 (1), Jan-Jun 2013, p. 23-28. 